\title{
EMBEDDING DERIVED CATEGORIES OF ENRIQUES SURFACES INTO DERIVED CATEGORIES OF FANO VARIETIES
}

\author{
ALEXANDER KUZNETSOV
}

\begin{abstract}
We show that the bounded derived category of coherent sheaves on a general Enriques surface can be realized as a semiorthogonal component in the derived category of a smooth Fano variety with diagonal Hodge diamond.
\end{abstract}

If a smooth projective variety $X$ over the field $\mathbb{C}$ of complex numbers has a full exceptional collection, then its Hodge diamond is diagonal, i.e.,

$$
h^{p, q}(X)=0 \quad \text { for } p \neq q .
$$

It is natural to ask whether the converse is true. A simple counterexample to this naive question is provided by an Enriques surface $S$ - its Hodge diamond looks like

$$
0{ }_{0}^{0}{ }_{1}^{1}{ }_{0}^{0} 0,
$$

so it is diagonal; on the other hand, its Grothendieck group $K_{0}(S)$ contains a 2-torsion class (see, for instance, GKMS13, Lemma 2.2]), hence the derived category cannot be generated by a full exceptional collection by the next simple lemma.

Lemma 1 (cf. [BP93, §3], GKMS13, Proposition 2.1(5)]). Let $\mathscr{T}$ be a triangulated category such that the Grothendieck group $K_{0}(\mathscr{T})$ contains a torsion class. Then $\mathscr{T}$ does not admit a full exceptional collection.

Proof. Assume $\mathscr{T}$ is generated by an exceptional collection of length $n$. Since the Grothendieck group is additive with respect to semiorthogonal decompositions, we have $K_{0}(\mathscr{T}) \cong \mathbb{Z}^{n}$. In particular, $K_{0}(\mathscr{T})$ is torsion free.

The question that is a bit less naive — whether a Fano variety with diagonal Hodge diamond necessarily has a full exceptional collection — was asked by Alexey Bondal back in 1989. This question was raised again in a recent paper [PS18. The main goal of this note is to show that the answer is still negative, and again counterexamples can be constructed using Enriques surfaces.

To be more precise, we construct a smooth Fano variety $X$ such that its bounded derived category $\mathbf{D}(X)$ of coherent sheaves has a semiorthogonal decomposition whose components are several exceptional objects and $\mathbf{D}(S)$, where $S$ is an Enriques surface. Thus, the Hodge diamond of $X$ is diagonal, but the Grothendieck group $K_{0}(X)$ contains a 2-torsion class (coming from $K_{0}(S)$ ), hence $\mathbf{D}(X)$ does not have a full exceptional collection by Lemma 1 .

In fact, we present two such constructions.

In the first construction, $S$ is a general Enriques surface from a certain divisorial family in the moduli space of Enriques surfaces — such $S$ are called "nodal Enriques surfaces" or "Reye congruences".

I was partially supported by the Russian Academic Excellence Project "5-100" and by the Program of the Presidium of the Russian Academy of Sciences 01 "Fundamental Mathematics and its Applications" under grant PRAS-18-01. I am grateful to A. Fonarev, D. Orlov and C. Shramov for inspiring discussions and the referee for useful comments. 
By [Cos83, Theorem 3.2.2] an Enriques surface $S$ of this type can be embedded into the Grassmannian $\operatorname{Gr}(2,4)$, and [IK15, Lemma 5.1] describes a resolution of its structure sheaf.

We consider the blowup

$$
M=\mathrm{Bl}_{S}(\operatorname{Gr}(2,4))
$$

Theorem 2. The variety $M$ is a Fano 4-fold with a semiorthogonal decomposition

$$
\mathbf{D}(M)=\left\langle\mathbf{D}(S), E_{1}, \ldots, E_{6}\right\rangle,
$$

where $E_{1}, \ldots, E_{6}$ are exceptional bundles. The Hodge diamond of $M$ is diagonal, but $K_{0}(M)$ contains a 2-torsion class; in particular $\mathbf{D}(M)$ does not have a full exceptional collection.

Proof. By [IK15, Lemma 5.2, 5.3] the variety $M$ can be embedded into the product $\operatorname{Gr}(2,4) \times \mathbb{P}^{3}$ as the zero locus of a regular section of the rank-3 vector bundle $S^{2} \mathscr{U}^{\vee} \otimes \mathscr{O}(1)$, where $\mathscr{U}$ is the tautological vector bundle of the Grassmannian. The determinant of this vector bundle is isomorphic to $\mathscr{O}(3) \otimes \mathscr{O}(3)$, hence by adjunction formula $\left.\omega_{M}^{-1} \cong(\mathscr{O}(1) \otimes \mathscr{O}(1))\right|_{M}$ is the restriction of an ample line bundle, hence $M$ is a Fano 4-fold.

The semiorthogonal decomposition is given by the Orlov's blowup formula and the fact that $\mathbf{D}(\operatorname{Gr}(2,4))$ is generated by an exceptional collection of length 6 . The Hodge diamond of $M$ looks like

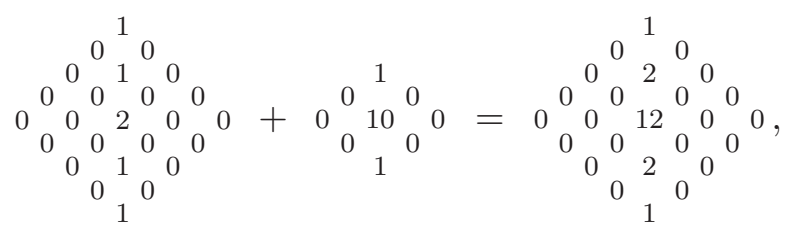

a combination of the Hodge diamonds of $\operatorname{Gr}(2,4)$ and $S$, again thanks to the blowup representation. The Grothendieck group is additive with respect to semiorthogonal decompositions, hence

$$
K_{0}(M)=K_{0}(S) \oplus \mathbb{Z}^{6}
$$

in particular the 2-torsion class in $S$ gives a 2-torsion class in $M$. We conclude by Lemma 1 ,

The second construction works for a general Enriques surface (i.e., corresponding to any point of an open subset in the moduli space of Enriques surfaces), at the price that the corresponding Fano variety is 6-dimensional.

Let $V_{1}$ and $V_{2}$ be a pair of 3-dimensional vector spaces. Consider the Veronese embeddings

$$
\mathbb{P}\left(V_{1}\right) \hookrightarrow \mathbb{P}\left(\mathrm{S}^{2} V_{1}\right) \hookrightarrow \mathbb{P}\left(\mathrm{S}^{2} V_{1} \oplus \mathrm{S}^{2} V_{2}\right), \quad \mathbb{P}\left(V_{2}\right) \hookrightarrow \mathbb{P}\left(\mathrm{S}^{2} V_{2}\right) \hookrightarrow \mathbb{P}\left(\mathrm{S}^{2} V_{1} \oplus \mathrm{S}^{2} V_{2}\right),
$$

and their join $\mathrm{J}\left(\mathbb{P}\left(V_{1}\right), \mathbb{P}\left(V_{2}\right)\right) \subset \mathbb{P}\left(\mathrm{S}^{2} V_{1} \oplus \mathrm{S}^{2} V_{2}\right)$. This is a singular 5-dimensional variety, whose singularities are resolved by the projective bundle

$$
\mathbf{J}:=\mathbb{P}_{\mathbb{P}\left(V_{1}\right) \times \mathbb{P}\left(V_{2}\right)}(\mathscr{O}(-2,0) \oplus \mathscr{O}(0,-2)) .
$$

Indeed, denote by $H_{1}$ and $H_{2}$ the pullbacks to $\mathbf{J}$ of the hyperplane classes of the two factors $\mathbb{P}\left(V_{1}\right)$ and $\mathbb{P}\left(V_{2}\right)$, by $H$ the Grothendieck relative class of the projectivization, and by $\pi: \mathbf{J} \rightarrow \mathbb{P}\left(V_{1}\right) \times \mathbb{P}\left(V_{2}\right)$ the projection. Then the natural embedding

$$
\mathscr{O}_{\mathbf{J}}(-H) \hookrightarrow \pi^{*}(\mathscr{O}(-2,0) \oplus \mathscr{O}(0,-2)) \hookrightarrow\left(\mathrm{S}^{2} V_{1} \otimes \mathscr{O}\right) \oplus\left(\mathrm{S}^{2} V_{2} \otimes \mathscr{O}\right)
$$

defines a morphism $\mathbf{J} \rightarrow \mathbb{P}\left(\mathrm{S}^{2} V_{1} \oplus \mathrm{S}^{2} V_{2}\right)$ which contracts the divisors

$$
\mathbb{P}_{\mathbb{P}\left(V_{1}\right) \times \mathbb{P}\left(V_{2}\right)}(\mathscr{O}(-2,0)) \subset \mathbf{J}, \quad \mathbb{P}_{\mathbb{P}\left(V_{1}\right) \times \mathbb{P}\left(V_{2}\right)}(\mathscr{O}(0,-2)) \subset \mathbf{J}
$$

onto the two Veronese surfaces $\mathbb{P}\left(V_{1}\right) \hookrightarrow \mathbb{P}\left(\mathrm{S}^{2} V_{1} \oplus \mathrm{S}^{2} V_{2}\right)$ and $\mathbb{P}\left(V_{2}\right) \hookrightarrow \mathbb{P}\left(\mathrm{S}^{2} V_{1} \oplus \mathrm{S}^{2} V_{2}\right)$, and takes the fibers of $\pi$ to the lines joining the corresponding points of these. 
Below we consider a global section of the vector bundle $\mathscr{O}_{\mathbf{J}}(H)^{\oplus 3}$ on $\mathbf{J}$. Note that

$$
H^{0}\left(\mathbf{J}, \mathscr{O}_{\mathbf{J}}(H)\right) \cong H^{0}\left(\mathbb{P}\left(V_{1}\right) \times \mathbb{P}\left(V_{2}\right), \mathscr{O}(2,0) \oplus \mathscr{O}(0,2)\right) \cong \mathrm{S}^{2} V_{1}^{\vee} \oplus \mathrm{S}^{2} V_{2}^{\vee},
$$

so such a section is given by a linear map

$$
\phi: W \rightarrow \mathrm{S}^{2} V_{1}^{\vee} \oplus \mathrm{S}^{2} V_{2}^{\vee}
$$

from a 3 -dimensional vector space $W$. We will denote the corresponding section also by $\phi$.

Lemma 3. The zero locus $S \subset \mathbf{J}$ of a general section $\phi$ of the vector bundle $\mathscr{O}_{\mathbf{J}}(H)^{\oplus 3}$ on $\mathbf{J}$ is an Enriques surface. A general Enriques surface can be obtained in this way.

Proof. Consider another projective bundle

$$
\widetilde{\mathbf{J}} \cong \mathbb{P}_{\mathbb{P}\left(V_{1}\right) \times \mathbb{P}\left(V_{2}\right)}(\mathscr{O}(-1,0) \oplus \mathscr{O}(0,-1)) .
$$

It is isomorphic to the blowup of $\mathbb{P}\left(V_{1} \oplus V_{2}\right)$ along the union of two skew planes $\mathbb{P}\left(V_{1}\right) \sqcup \mathbb{P}\left(V_{2}\right) \subset \mathbb{P}\left(V_{1} \oplus V_{2}\right)$ with the exceptional divisors

$$
E_{1}=\mathbb{P}_{\mathbb{P}\left(V_{1}\right) \times \mathbb{P}\left(V_{2}\right)}(\mathscr{O}(-1,0)) \subset \widetilde{\mathbf{J}}, \quad E_{2}=\mathbb{P}_{\mathbb{P}\left(V_{1}\right) \times \mathbb{P}\left(V_{2}\right)}(\mathscr{O}(0,-1)) \subset \widetilde{\mathbf{J}} .
$$

Denote by $\tilde{H}_{1}$ and $\tilde{H}_{2}$ the pullbacks to $\widetilde{\mathbf{J}}$ of the hyperplane classes of the two factors $\mathbb{P}\left(V_{1}\right)$ and $\mathbb{P}\left(V_{2}\right)$, and by $\tilde{H}$ the Grothendieck relative class of the projectivization. Then $E_{1} \equiv \tilde{H}-\tilde{H}_{2}$ and $E_{2} \equiv \tilde{H}-\tilde{H}_{1}$.

Consider the involution of the bundle $\mathscr{O}(-1,0) \oplus \mathscr{O}(0,-1)$ acting with weight -1 on the first summand and with weight 1 on the second, and the corresponding involution $\tau$ of $\widetilde{\mathbf{J}}$. The fixed locus of $\tau$ is the union of the exceptional divisors $E_{1} \sqcup E_{2}$, and the quotient $\widetilde{\mathbf{J}} / \tau$ is isomorphic to $\mathbf{J}$ with the quotient map $f: \widetilde{\mathbf{J}} \rightarrow \mathbf{J}$ induced by the projection

$$
\mathrm{S}^{2}(\mathscr{O}(-1,0) \oplus \mathscr{O}(0,-1))=\mathscr{O}(-2,0) \oplus \mathscr{O}(-1,-1) \oplus \mathscr{O}(0,-2) \rightarrow \mathscr{O}(-2,0) \oplus \mathscr{O}(0,-2) .
$$

Note also, that $\mathscr{O}_{\widetilde{\mathbf{J}}}(2 \tilde{H}) \cong f^{*}\left(\mathscr{O}_{\mathbf{J}}(H)\right)$, and this induces an isomorphism

$$
H^{0}\left(\widetilde{\mathbf{J}}, \mathscr{O}_{\widetilde{\mathbf{J}}}(2 \tilde{H})\right)^{\tau} \cong H^{0}\left(\mathbf{J}, \mathscr{O}_{\mathbf{J}}(H)\right) \cong \mathrm{S}^{2} V_{1}^{\vee} \oplus \mathrm{S}^{2} V_{2}^{\vee}
$$

between the space of $\tau$-invariant global sections of $\mathscr{O}_{\widetilde{\mathbf{J}}}(2 \tilde{H})$ and the space of global sections of $\mathscr{O}_{\mathbf{J}}(H)$. Therefore, the preimage

$$
\tilde{S}:=f^{-1}(S) \subset \widetilde{\mathbf{J}}
$$

is the zero locus of a general $\tau$-invariant section of the vector bundle $\mathscr{O}_{\mathbf{J}}(2 \tilde{H})^{\oplus 3}$. We have

$$
K_{\tilde{S}} \equiv K_{\widetilde{\mathbf{J}}}+6 \tilde{H} \equiv\left(-3 \tilde{H}_{1}-3 \tilde{H}_{2}\right)+\left(\tilde{H}_{1}+\tilde{H}_{2}-2 \tilde{H}\right)+6 \tilde{H} \equiv 4 \tilde{H}-2 \tilde{H}_{1}-2 \tilde{H}_{2} \equiv 2 E_{1}+2 E_{2} .
$$

Recall that $S$ is defined by a map $\phi: W \rightarrow \mathrm{S}^{2} V_{1}^{\vee} \oplus \mathrm{S}^{2} V_{2}^{\vee}$. Clearly $\tilde{S} \cap E_{i}$ is equal to the intersection of three conics in $\mathbb{P}\left(V_{i}\right)$ corresponding to the induced map $\phi_{i}: W \rightarrow \mathrm{S}^{2} V_{i}^{\vee}$, hence is empty for a general choice of $S$. This shows that for a general $S$, the surface $\tilde{S}$ meets neither $E_{1}$ nor $E_{2}$, hence $K_{\tilde{S}} \equiv 0$.

Furthermore, it is easy to see that $H^{1}\left(\tilde{S}, \mathscr{O}_{\tilde{S}}\right)=0$ (for instance, by using the Koszul resolution of $\mathscr{O}_{\tilde{S}}$ on $\widetilde{\mathbf{J}})$, hence $\tilde{S}$ is a K3 surface. As $\tilde{S}$ does not intersect the fixed locus $E_{1} \sqcup E_{2}$ of $\tau$, the involution $\tau$ acts freely on $\tilde{S}$, hence

$$
S \cong \tilde{S} / \tau \subset \widetilde{\mathbf{J}} / \tau=\mathbf{J}
$$

is an Enriques surface.

Finally, note that the surface $\tilde{S}$ defined above coincides with the surface $X$ in [Bea96, Exercise VIII.18], and the involution $\tau$ on $\tilde{S}$ coincides with the involution $\sigma$ in loc. cit. Therefore, the quotient $S=\tilde{S} / \tau$ is a general Enriques surface. 
Next we consider the product $\mathbf{J} \times \mathbb{P}(W)$ that parametrizes the linear system of sections of $\mathscr{O}_{\mathbf{J}}(H)$, cutting out $S$ in $\mathbf{J}$. Denote by $H^{\prime}$ the hyperplane class of this $\mathbb{P}(W)$ and let

$$
X \subset \mathbf{J} \times \mathbb{P}(W)
$$

be the universal divisor from the linear system, i.e., the zero locus on $\mathbf{J} \times \mathbb{P}(W)$ of the global section of the line bundle $\mathscr{O}_{\mathbf{J}}(H) \otimes \mathscr{O}\left(H^{\prime}\right)$ corresponding to the map $\phi$.

Theorem 4. The variety $X$ is a Fano 6-fold with a semiorthogonal decomposition

$$
\mathbf{D}(X)=\left\langle\mathbf{D}(S), F_{1}, \ldots, F_{36}\right\rangle,
$$

where $F_{1}, \ldots, F_{36}$ are exceptional bundles. The Hodge diamond of $X$ is diagonal, but $K_{0}(X)$ contains a 2-torsion class; in particular $\mathbf{D}(X)$ does not have a full exceptional collection.

Proof. The canonical class of $X$ is equal to

$K_{X}=K_{\mathbf{J}}+K_{\mathbb{P}(W)}+\left(H+H^{\prime}\right)=\left(-3 H_{1}-3 H_{2}\right)+\left(2 H_{1}+2 H_{2}-2 H\right)-3 H^{\prime}+\left(H+H^{\prime}\right)=-H_{1}-H_{2}-H-2 H^{\prime}$.

Let us show that $-K_{X}$ is ample. Clearly, for this it is enough to check that $H+H_{1}+H_{2}$ is ample on $\mathbf{J}$. By [Har66, Proposition 3.2] this is equivalent to ampleness of its pushforward $\mathscr{O}\left(3 H_{1}+H_{2}\right) \oplus \mathscr{O}\left(H_{1}+3 H_{2}\right)$ on $\mathbb{P}\left(V_{1}\right) \times \mathbb{P}\left(V_{2}\right)$, which follows from [Har66, Proposition 2.2] and from ampleness of the summands. We conclude that $X$ is a Fano 6-fold.

The map $X \rightarrow \mathbf{J}$ has general fiber $\mathbb{P}^{1}$, and over the surface $S \subset \mathbf{J}$ the fibers jump to $\mathbb{P}^{2}$. Therefore,

$$
\mathbf{D}(X)=\langle\mathbf{D}(S), \mathbf{D}(\mathbf{J}), \mathbf{D}(\mathbf{J})\rangle,
$$

either by [Kuz07, Theorem 8.8], or by Orl06, Proposition 2.10]. Since $\mathbf{J}$ is a $\mathbb{P}^{1}$-bundle over $\mathbb{P}^{2} \times \mathbb{P}^{2}$, its derived category is generated by $3 \cdot 3 \cdot 2=18$ exceptional bundles, hence we obtain the required semiorthogonal decomposition for $\mathbf{D}(X)$. Finally, the Hodge diamond of $X$ looks like

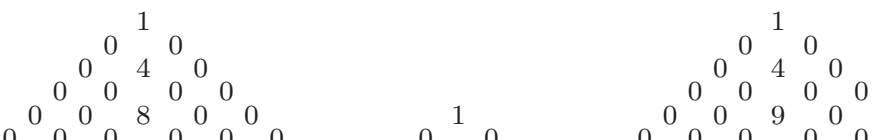

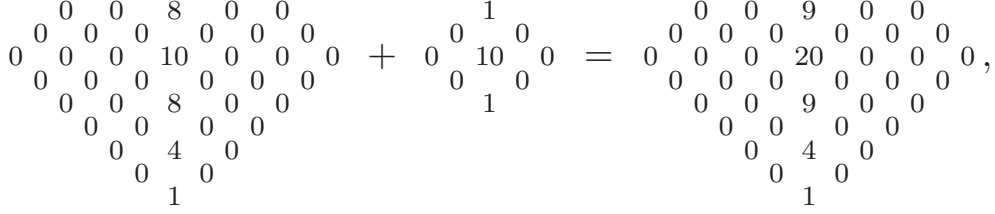

a combination of the Hodge diamonds of $\mathbf{J} \times \mathbb{P}^{1}$ and $S$. The Grothendieck group is additive with respect to semiorthogonal decompositions, hence

$$
K_{0}(X)=K_{0}(S) \oplus \mathbb{Z}^{36}
$$

in particular the 2-torsion class in $S$ gives a 2-torsion class in $X$. We conclude by Lemma 1 ,

Remark 5. The embedding of the derived category of a general Enriques surface into a Fano variety, constructed in Theorem 4, solves for them so-called "Fano-visitor problem" suggested by Alexey Bondal in 2011, see [BBF16, KKLL17, KL15, Nar17, FK18]. Note that a similar embedding of $\mathbf{D}(S)$ into the derived category of a Fano orbifold was constructed in KL15, 6.2.3].

\section{REFERENCES}

[BBF16] Marcello Bernardara, Michele Bolognesi, and Daniele Faenzi. Homological projective duality for determinantal varieties. Adv. Math., 296:181-209, 2016.

[Bea96] Arnaud Beauville. Complex algebraic surfaces, volume 34 of London Mathematical Society Student Texts. Cambridge University Press, Cambridge, second edition, 1996. Translated from the 1978 French original by R. Barlow, with assistance from N. I. Shepherd-Barron and M. Reid. 
[BP93] A. I. Bondal and A. E. Polishchuk. Homological properties of associative algebras: the method of helices. Izv. Ross. Akad. Nauk Ser. Mat., 57(2):3-50, 1993.

[Cos83] François R. Cossec. Reye congruences. Trans. Amer. Math. Soc., 280(2):737-751, 1983.

[FK18] Anton Fonarev and Alexander Kuznetsov. Derived categories of curves as components of fano manifolds. Journal of the LMS, 97(1):24-46, 2018.

[GKMS13] Sergey Galkin, Ludmil Katzarkov, Anton Mellit, and Evgeny Shinder. Minifolds and phantoms. arXiv preprint arXiv:1305.4549, 2013.

[Har66] Robin Hartshorne. Ample vector bundles. Inst. Hautes Études Sci. Publ. Math., (29):63-94, 1966.

[IK15] Colin Ingalls and Alexander Kuznetsov. On nodal Enriques surfaces and quartic double solids. Math. Ann., 361(1-2):107-133, 2015.

[KKLL17] Young-Hoon Kiem, In-Kyun Kim, Hwayoung Lee, and Kyoung-Seog Lee. All complete intersection varieties are Fano visitors. Adv. Math., 311:649-661, 2017.

[KL15] Young-Hoon Kiem and Kyoung-Seog Lee. Fano visitors, Fano dimension and orbifold Fano hosts. ArXiv e-prints, April 2015.

[Kuz07] Alexander Kuznetsov. Homological projective duality. Publ. Math. Inst. Hautes Études Sci., (105):157-220, 2007.

[Nar17] M. S. Narasimhan. Derived categories of moduli spaces of vector bundles on curves. J. Geom. Phys., 122:53-58, 2017.

[Orl06] D. O. Orlov. Triangulated categories of singularities, and equivalences between Landau-Ginzburg models. Mat. Sb., 197(12):117-132, 2006.

[PS18] V. Przyjalkowski and C. Shramov. Hodge complexity for weighted complete intersections. arXiv:1801.10489 January 2018.

Algebraic Geometry Section, Steklov Mathematical Institute of Russian Academy of Sciences, 8 Gubkin str., Moscow 119991 Russia

The Poncelet Laboratory, Independent University of Moscow

Laboratory of Algebraic Geometry, National Research University Higher School of Economics, RUSSIAN FEDERATION

E-mail address: akuznet@mi-ras.ru 